\title{
ANALOGUE OF PONTRYAGIN CHARACTER THEORY FOR TOPOLOGICAL SEMIGROUPS
}

\author{
THOMAS BOWMAN
}

\begin{abstract}
The character semigroups of a wide class of abelian continuous inverse semigroups are determined. A duality theorem is given for which a special case gives necessary and sufficient conditions for duality to hold for locally compact semigroups.
\end{abstract}

For the semigroup analogue of Pontryagin character theory of groups, replace the circle group by the multiplicative semigroup $D$ of complex numbers with modulus less than or equal to one. If $S$ is a topological abelian semigroup, then a semicharacter of $S$ will be a continuous homomorphism of $S$ into $D$ which is not identically zero. If $S$ has an identity, then the set $S^{\wedge}$ of all semicharacters of $S$ endowed with pointwise multiplication and the compact-open topology is a topological abelian semigroup. These concepts were first studied by C.W. Austin in [1]. He classified the discrete semigroups for which duality holds as abelian inverse semigroups with identity. If the results of [1] and [9], or [1], [2] and [3] are combined, we find that the compact semigroups for which duality holds are the abelian inverse semigroups with identity whose idempotent sets are totally disconnected. In [2], it was shown that a necessary condition for duality to hold is that $S$ be an abelian inverse semigroup with identity and continuous inversion.

For $S$ an abelian inverse semigroup with identity, this paper will give an explicit description, both algebraic and topological, of $S^{\wedge}$. Although there will be some restrictions on $S$, this approach will be general enough to cover the case where the semilattice $E(S)$ of idempotents of $S$ is locally compact Lawson and inversion is continuous. Necessary and sufficient conditions are then given for duality to hold for an inverse semigroup in terms of its semilattice of idempotents and $\mathcal{H}$-classes.

1. Preliminaries. The algebraic structure of inverse semigroups whose set of idempotents is a semilattice was determined by A. H. Clifford [6, p. 128]. The author in [4] showed the relationship of compact inverse

Presented to the Society, November 24, 1972; received by the editors January 12, 1973. AMS (MOS) subject classifications (1970). Primary 22A25; Second ary 20M30, $43 A 40,43 A 65,43 A 95$. 
semigroups whose set of idempotents is a Lawson semilattice to inverse limit-preserving functors. It will be necessary for this paper to restate Clifford's work in functorial language. We will restrict our attention to the abelian case. Note that for abelian semigroups the following three classes are the same: semigroups which are a union of groups, semigroups which are a semilattice of groups and inverse semigroups. If $X$ is a semilattice, then $X$ may be considered a category by defining $x \rightarrow y$ iff $x \geq y$. For $G$ a functor from $X$ into the category of abelian groups, denote $G(x \rightarrow y)$ by $G_{y x}$ for $x \geq y \in$ $X$. Let $\mathrm{Cl}(X, G)$ be the disjoint union of $G(x), x \in X . \mathrm{Cl}(X, G)$ will be made into an abelian inverse semigroup by defining for $s \in G(x), t \in G(y)$ and $u=x y$, st $=G_{u x}(s) \cdot G_{u y}(t)$. Conversely, if $S$ is an abelian inverse semigroup and $X=E(S)$, define $G(x)=\mathcal{H}(x)$ and for $x \geq y$ define $G_{y x}: G(x)$ $\rightarrow G(y)$ by $G_{y x}(s)=s y$ for $s \in G(x)$. Then $G$ is a functor from $X$ into the category of abelian groups and $S$ is algebraically isomorphic to $\mathrm{Cl}(X, G)$. The functor $G$ is called the associated functor of $S$. If $S$ happens to be a topological semigroup we will ignore for a moment the topologies on these sets.

1.1. Definition. A topological semilattice $X$ is said to have the lower extension property (LEP) if for any open-closed prime ideal $P$ of $X$ and any element $x \notin P$ there exists a $z \notin P$ such that $x \in U(z)^{\circ}$ where $U(z)=$ $\{y \in X: y \geq z\}$.

An interesting class of locally compact semilattices which have the lower extension property is the class of Lawson semilattices: each element has a fundamental system of open neighborhoods which are semilattices [8]. Fulp [7] gave the following definition.

1.2. Definition. If $E$ is a topological semilattice, we shall say that $E$ satisfies the separation hypothesis $(\mathrm{SH})$ iff whenever $C \subseteq E$ is compact and $C \cap P=\square$ for some open-closed prime ideal $P$ of $E$, then there exists $e \in E$ such that $C \subseteq U(e)$ and $U(e) \cap P=\square$.

1.3. Proposition. If $X$ is a topological semilattice, then LEP implies $S H$. If in addition $X$ is locally compact, $S H$ implies LEP.

Proof. Let $C$ be a compact set and $P$ an open-closed prime ideal with $C \cap P=\square$. For each element $c \in C$, there exists a $c^{\prime} \in X-P$ such that $c \in U\left(c^{\prime}\right)^{\circ}$. The collection $U\left(c^{\prime}\right)^{\circ}, c \in C$, is an open covering of $C$ so there exists a finite subcovering which will be denoted by $U\left(c_{1}^{\prime}\right)^{\circ}, \ldots$, $U\left(c_{n}^{\prime}\right)^{\circ}$. Let $e=c_{1}^{\prime} c_{2}^{\prime} \cdots c_{n}^{\prime}$. Since $P$ is prime, $e \notin P$ and $C \subseteq U(e)$.

Now, suppose that $X$ is locally compact and satisfies the separation hypothesis. If $P$ is an open-closed prime ideal and $x \in X-P$, there exists 
an open neighborhood $\mathcal{U}$ of $x$ such that $\mathcal{U}^{-}$is compact and $\mathcal{U}^{-} \cap P=\square$. By $\mathrm{SH}$, there exists a $z \in X$ such that $\mathcal{U}^{-} \subseteq U(z)$ and $U(z) \cap P=\square$. Therefore, $z \notin P$ and $x \in U(z)^{\circ}$.

Notation. If $S$ and $T$ are topological semigroups and $K$ is a subset of $S$ and $\mathcal{U}$ is a subset of $T$, then $(K, \mathcal{U})=\{f \in \operatorname{Hom}(S, T): f(K) \subset \mathcal{U}\}$. Recall that the collection of all sets $(K, \mathcal{U})$, where $K$ is compact and $\mathcal{U}$ is open, is a subbasis for the compact-open topology on $\operatorname{Hom}(S, T)$.

If $S$ is an inverse semigroup and $\phi \in S^{\wedge}$, then $|\phi(s)|=0$ or $|\phi(s)|=1$ for $s \in S$. Define $N(\phi)=\{s \in S: \phi(s)=0\}$, and $I(\phi)=\{s \in S:|\phi(s)|=1\}$.

1.4. Lemma. If $X$ is a topological semilattice with $L E P$, define $\mathfrak{U}=$ $\{(\{u\},\{1\}): u \in X\}$ and $\mathbb{B}=\{(K,\{0\}) \cap L: K$ a compact subset of $X$ and $L \in$ $\mathfrak{U}\}$. Then $\mathbb{U} \cup \mathbb{B}$ is a basis for the compact-open topology on $X$.

Proof. Suppose $\phi \in X^{\wedge}$ and $\phi$ is not the identity of $\lambda^{\wedge}$. If $W$ is an open neighborhood of $\phi$, then there exist compact subsets of $X, K_{1}, \cdots, K_{n}$ and $L_{1}, \cdots, L_{m}$ such that $\phi \in\left(K_{1},\{0\}\right) \cap \cdots \cap\left(K_{n},\{0\}\right) \cap\left(L_{1},\{1\}\right) \cap \cdots$ $\cap\left(L_{m},\{1\}\right) \subset W$. Letting $K=K_{1} \cup \cdots \cup K_{n}$ and $L=L_{1} \cup \cdots \cup L_{m}$, we have $\phi \in(K,\{0\}) \cap(L,\{1\}) \subset W$. By 1.3,X satisfies the separation hypothesis, so there exists $u \in X$ such that $L \subseteq U(u)$ and $U(u) \cap N(\phi)=\square . L \subseteq$ $U(u)$ implies $(\{u\},\{1\}) \subset(L,\{1\})$. Whence

$$
\phi \in(K,\{0\}) \cap(\{u\},\{1\}) \subset W .
$$

It is clear from the above that $U$ is a fundamental system of neighborhoods for the identity of $X^{\wedge}$.

Convention. It will be the general practice in this paper to identify things which are naturally isomorphic. For example, a locally compact abelian group will be identified to its second character group.

2. Character semigroups. In this section, $S$ will be an abelian continuous inverse semigroup with identity whose semilattice of idempotents $X$ has the lower extension property. Note that the continuity of inversion implies the map $p: S \rightarrow E(S)$ that sends each element of $S$ into the unique idempotent in its $\mathcal{H}$-class is continuous. Let $G$ be the associated functor of $S$; we will define a functor $G^{\wedge}$ on $X^{\wedge}$ that will be the associated functor of $S^{\wedge}$. For $x \geq y \in X$, define the map $G_{y x}^{\wedge}: G(y)^{\wedge} \rightarrow G(x)^{\wedge}$ by $G_{y x}^{\wedge}(\phi)=$ $\phi \circ G_{y x}$ for $\phi \in G(y)^{\wedge}$. For $\epsilon \in X^{\wedge}$, define

$$
\begin{aligned}
& \hat{G^{\wedge}}(\epsilon)=\operatorname{inv} \lim \left\{G(x)^{\wedge}, G_{y x}^{\wedge}: x \geq y \in I(\epsilon)\right\} \\
& =\left\{\langle\omega\rangle \in \Pi \hat{G_{x}}: \hat{G_{y x}}\left(\omega_{y}\right)=\omega_{x} \text { for } x \geq y \in I(\epsilon)\right\} \text {. }
\end{aligned}
$$


If $\epsilon, \delta$ in $\mathrm{X}^{\wedge}$, then $\epsilon \geq \delta$ iff $I(\epsilon) \supset I(\delta)$. So the natural projection map of $\Pi G(x)^{\wedge}, x \in I_{\epsilon}()$, onto $\Pi G(x)^{\wedge}, x \in I(\delta)$, carries $G^{\wedge}(\epsilon)$ into $G^{\wedge}(\delta)$. Define $G_{\hat{\delta \epsilon}}: G^{\wedge}(\epsilon) \rightarrow G^{\wedge}(\delta)$ to be the restriction of this projection map. Then $G^{\wedge}$ is a functor from $X^{\wedge}$ into the category of topological abelian groups.

2.1. Theorem. If $S$ is an abelian continuous-inverse semigroup with identity whose semilattice $X$ of idempotents has LEP and if $G$ is the associated functor of $S$, then $S^{\wedge}$ is isomorphic to $\mathrm{Cl}\left(X^{\wedge}, G^{\wedge}\right)$.

Proof. If $\epsilon \in E\left(S^{\wedge}\right)$, let $\epsilon_{X}=\epsilon \mid X$. The continuity of inversion implies that the map $\epsilon \rightarrow \epsilon_{X}$ is an isomorphism of $E\left(S^{\wedge}\right)$ onto $X \hat{\text {. For }} k \in X$ and $\phi$ $\epsilon S^{\hat{\imath}}$, let $\phi_{k}=\phi \mid G(k)$. If $\epsilon \in E\left(S^{\wedge}\right)$, then $\phi \in \mathcal{H}(\epsilon)$ iff $I(\phi)=I(\epsilon)$. For $x \geq y$ $\epsilon I(\phi) \cap X, \phi_{x}=\phi_{y}{ }^{\circ} G_{y x}=G_{y x}^{\hat{x}}\left(\phi_{y}\right)$. For each $\epsilon \in E\left(s^{\wedge}\right)$, define $\pi_{\epsilon}: \mathcal{H}(\epsilon)$ $\rightarrow G^{\wedge}\left(\epsilon_{X}\right)$ by $\pi_{\epsilon}(\phi)=\left\langle\phi_{x}\right\rangle, x \in I(\phi) \cap X=I\left(\epsilon_{X}\right)$. It is straightforward that $\pi_{\epsilon}$ is an injective homomorphism. It remains to show that $\pi_{\epsilon}$ is onto. Suppose $\left\langle\omega_{x}\right\rangle \in G^{\wedge}\left(\epsilon_{X}\right)$. Define $\omega: S \rightarrow D$ by

$$
\begin{array}{rlrl}
\omega(s) & =\omega_{x}(s) & & \text { if } s \in \mathcal{H}(x) \text { and } x \in I\left(\epsilon_{X}\right), \\
& =0 & \text { if } s \in \mathcal{H}(x) \text { and } x \in N\left(\epsilon_{X}\right) .
\end{array}
$$

It is easily seen that $\omega$ is a homomorphism. It must be shown that $\omega$ is continuous. Let $\left\{s_{\alpha}\right\}, a \in \Lambda$, be a net in $S$ such that $\lim s_{\alpha}=s \in S$. If $p(s) \in N\left(\epsilon_{X}\right)$, then $\omega(s)=0$. Since $N\left(\epsilon_{X}\right)$ is open, there exists an $\alpha_{0} \in \Lambda$ such that for $\beta \geq a_{0}, p\left(s_{\beta}\right) \in N\left(\epsilon_{X}\right)$, but this implies $\omega\left(s_{\beta}\right)=0$. Whence $\lim \omega\left(s_{\alpha}\right)=0=\omega(s)$. If $p(s) \notin N\left(\epsilon_{X}\right)$, by LEP there exists a $z \in X$ such that $z \notin N\left(\epsilon_{X}\right)$ and $p(s) \in U(z)^{\circ}$. There exists an $\alpha_{1} \in \Lambda$ such that for $\beta \geq$ $\alpha_{1}, p\left(s_{\beta}\right) \in U(z)^{\circ} \subseteq I\left(\epsilon_{X}\right)$. For $t \in U(z)$ and $u=p(t), \quad \omega(t)=\omega_{u}(t)=$ $\left[G_{z u}^{\hat{y}}\left(\omega_{z}\right)\right](t)=\omega_{z} \circ G_{z u}(t)=\omega_{z}(z t)$. It follows that $\lim \omega\left(s_{\alpha}\right)=\lim \omega_{z}\left(z s_{\alpha}\right)=$ $\omega_{z}(z s)=\omega(s)$. Thus $\omega$ is continuous.

Define $\Re_{:} S^{\wedge} \rightarrow \mathrm{Cl}\left(X^{\wedge}, G^{\wedge}\right)$ by $\Re(\phi)=\Re_{\epsilon}(\phi)$ for $\phi \in \mathcal{H}(\epsilon)$. It is clear that $\pi$ is bijective. If $\phi \in \mathcal{H}(\epsilon), \psi \in \mathcal{H}(\delta)$ and $\mu=\epsilon \delta$, then

$$
\begin{aligned}
\Re(\phi \psi) & =\Re(\mu \phi \mu \psi)=\Re_{\mu}(\mu \phi) \Re_{\mu}(\mu \psi) \\
& =\hat{G_{\mu \epsilon}}\left(\Re_{\epsilon}(\phi)\right) \cdot \hat{G_{\mu \delta}}\left(\Re_{\delta}(\psi)\right)=\Re_{\epsilon}(\phi) \cdot \Re_{\delta}(\psi)=\Re_{(\phi)} \Re_{(}(\psi) .
\end{aligned}
$$

Remark. From the proof we see that the group $G^{\wedge}(\epsilon)$ consists precisely of those points $\left\langle\omega_{x}\right\rangle$ in $\Pi\left\{G(x)^{\wedge}: x \in I(\epsilon) \cap X\right\}$ such that for some $\phi \in \mathcal{H}(\epsilon)$ we have $\omega_{x}=\phi \mid G(x)$ for all $x \in I(\epsilon) \cap X$. When it seems expedient we will identify $\left\langle\omega_{x}\right\rangle$ with $\phi$.

If we identify $S^{\wedge}$ and $\mathrm{Cl}\left(X^{\wedge}, G^{\wedge}\right)$ by the isomorphism of the preceding theorem, the next theorem gives a basis for the compact-open topology on $\mathrm{Cl}\left(X^{\wedge}, G^{\wedge}\right)$. 
2.2. Theorem. For $\mathrm{Cl}\left(X^{\wedge}, G^{\wedge}\right)$, let $\mathcal{U}$ be an open set of $X^{\wedge}, k \in X$, and

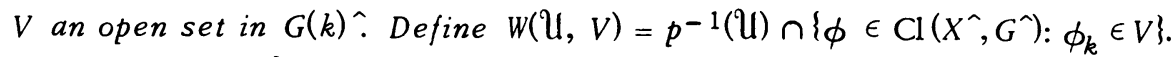
Sets of the form $W(\mathcal{U}, V)$ are a basis for the compact-open topology on $\mathrm{Cl}\left(X^{\wedge}, G^{\wedge}\right)$.

Proof. All sets $W(\mathcal{U}, V)$ are open in the compact-open topology. So it remains to show that for $\phi \in \mathrm{Cl}\left(X^{\wedge}, G^{\wedge}\right)$ and $A$ an open neighborhood of $\phi$, there exists a set of the form $W(\mathcal{U}, V)$ such that $\phi \epsilon$ $W(\mathcal{U}, V) \subset A$. There exist compact sets $K_{1}, \ldots, K_{n}$ and $L_{1}, \cdots, L_{m}$ of $S$ and open sets $\mathcal{U}_{1}, \ldots, \mathcal{U}_{m}$ of $\{z \in \mathrm{C}:|z|=1\}$ such that $\phi \epsilon$ $\left(K_{1},\{0\}\right) \cap \ldots \cap\left(K_{n},\{0\}\right) \cap\left(L_{1}, \mathcal{U}_{1}\right) \cap \ldots \cap\left(L_{m}, \mathcal{U}_{m}\right) \subset A$. Let $K=$ $p\left(K_{1} \cup \cdots \cup K_{n}\right)$ and $L=p\left(L_{1} \cup \cdots \cup L_{m}\right)$. Since $p$ is continuous, $(K,\{0\})$ $\cap(L,\{1\})$ is an open neighborhood of $\phi$ in the compact-open topology.

Since $X$ satisfies the separation hypothesis, there exists a $k \in X$ such that $k \notin N(\phi)$ and $L \subset U(k)$. For $1 \leq i \leq m, \phi \in\left(k L_{i}, \mathcal{U}_{i}\right) \subset\left(L_{i}, \mathcal{U}_{i}\right)$. Let $\mathcal{U}=(K,\{0\}) \cap(\{k\},\{1\})$ and let $V=\left(k L_{1}, \mathcal{U}_{1}\right) \cap \ldots \cap\left(k L_{m}, \mathcal{U}_{m}\right)$, then $\phi \epsilon$ $w(\mathcal{U}, V) \subset A$.

The following corollary removes the condition of local compactness from Theorem 3 by Fulp [7]. By 1.3, it is apparently a slight generalization of his result.

Corollary. Let $S$ be an abelian continuous-inverse semigroup with identity, whose semilattice of idempotents $X$ has the lower extension property. If $\epsilon \in E\left(S^{\wedge}\right)$, then $\mathcal{H}(\epsilon)$ is iseomorphic to $G^{\wedge}\left(\epsilon_{X}\right)=\operatorname{inv} \lim \left\{G(x) \hat{\wedge}, G_{y x}: x \geq y \epsilon\right.$ $I(\epsilon) \cap X\}$.

Proof. By 2.1 and 2.2, $\mathcal{H}(\epsilon)$ is iseomorphic to $G^{\wedge}\left(\epsilon_{X}\right)=\operatorname{inv} \lim \left\{G(x)^{\wedge}, G_{y x}\right.$ : $x \geq y \in I(\epsilon) \cap X\}$ in the topology induced from $\mathrm{Cl}\left(X^{\wedge}, G^{\wedge}\right)$. In $\Pi G(x) \wedge, x \epsilon$ $I(\epsilon) \cap X$, sets of the form $\mathcal{U}_{k}=\left\{\phi: \phi_{k} \in \mathcal{U}\right\}$, where $k \in I(\epsilon) \cap X$ and $\mathcal{U}$ open in $G(k)^{\wedge}$, are a subbasis for the product topology. Since $\mathcal{U}_{k} \cap G^{\wedge}\left(\epsilon_{X}\right)=\{\phi \epsilon$ $\left.G^{\wedge}\left(\epsilon_{X}\right): \phi_{k} \in \mathcal{U}\right\}$, the topologies induced from the product and from $\mathrm{Cl}\left(X^{\wedge}, G^{\wedge}\right)$ coincide.

3. Duality. For $S$ a topological semigroup with identity and $s \in S$ define $\tilde{s} \in S^{\wedge}$ by $\tilde{s}(\phi)=\phi(s)$ for all $\phi \in S^{\wedge}$. The map $s \rightarrow \tilde{s}: S \rightarrow S^{\wedge}$ is a continuous homomorphism.

3.1 Definition [3]. An element $e$ of a topological semilattice $X$ is said to be generating if $J_{e}=\{f \in X: f e \neq e\}$ is an open-closed prime ideal. Denote the set of all generating elements by $X_{g}$. If $e \in X_{g}$, define $\epsilon_{e} \epsilon$ $X^{\wedge}$ by

$$
\begin{aligned}
\epsilon_{e}(z) & =1, \quad z \geq e, \\
& =0, \quad z \geq e .
\end{aligned}
$$


3.2. Definition. A topological semilattice $X$ is said to be strongly separated (SS) if for every open-closed prime ideal $P$ and for each element $x$ $\in X-P$ there exists a $z \in X_{g}$ such that $z \notin P$ and $x \in U(z)$.

Remark. Any locally compact totally disconnected semilattice is strongly separated [3]. Also, note that SS implies LEP.

3.3. Lemma. If $X$ is a strongly separated semilattice and $x \rightarrow \tilde{x}$ : $X \rightarrow X^{\wedge}$ is an iseomorphism, then $X^{\wedge}$ is strongly separated.

Proof. Suppose $R$ is an open-closed prime ideal of $X^{\wedge}$ and $\phi \in X^{\wedge}-$ $R$. Since $x \rightarrow \tilde{x}: X \rightarrow X^{\wedge}$ is onto, there exists an $x \in X$ such that $N(\tilde{x})=$ $R$. Whence $\phi(x)=1$ and $x \in X-N(\phi)$. Since $X$ is strongly separated, there exists a $z \in X_{g}$ such that $x \in U(z)$ and $U(z) \cap N(\phi)=\square$. Since $x \in$ $U(z), \epsilon_{z}(x)=1$ which implies $\epsilon_{z} \in X^{\wedge}-R$. Now $I\left(\epsilon_{z}\right)=U(z) \subset I(\phi)$. Hence $\epsilon_{z} \leq \phi$. If $J=\left\{\psi \in X^{\wedge}: \psi \epsilon_{z} \neq \epsilon_{z}\right\}$, then $J=N(\tilde{z})$. It follows that $J$ is an open-closed prime ideal.

Notation. Let $\mathcal{P}$ denote the full subcategory of abelian topological groups for which the Pontryagin duality theorem holds.

The following theorem is related to [3, Theorem 2.6] and [7, Theorem $4]$.

3.4. Theorem. If $X$ is a strongly separated semilattice such that $x \rightarrow$ $\tilde{x}: X \rightarrow X^{\wedge \wedge}$ is an iseomorphism and $G$ is a functor from $X$ into $\mathcal{P}$, then the statements $\mathrm{A}$ and $\mathrm{B}$ are equivalent.

A. $S=\mathrm{Cl}(X, G)$ has a unique topology with the following properties:

(i) $S$ is a continuous-inverse semigroup.

(ii) The topologies of $X$ and $G(x), x \in X$, coincide with the subspace topologies.

(iii) The map $s \rightarrow \tilde{s}: S \rightarrow S^{\wedge \wedge}$ is an iseomorphism.

B. For $x \in X$, the map $\psi_{x}$ of $G(x)$ into inv $\lim \left\{G(y), G_{z y}: z \leq y \leq x\right.$ and $\left.z, y \in X_{g}\right\}$ defined by $\psi_{x}(g)=\left\langle G_{y x}(g)\right\rangle, y \in X_{g}$, is an iseomorphism.

Proof. Suppose $S=\mathrm{Cl}(X, G)$ has a topology for which it is a continuous inverse semigroup and which realizes the topologies of $X$ and $G(x)$, $x \in X$. By 3.3, $X^{\wedge}$ is SS which implies $X^{\wedge}$ has LEP. From 2.1 and 2.2, $S^{\wedge}$ $=\mathrm{Cl}\left(X^{\wedge}, G^{\wedge}\right)$ and $S^{\wedge \wedge}=\mathrm{Cl}\left(X^{\wedge \wedge}, G^{\wedge \wedge}\right)$. If $x \in X$ and $\epsilon \in I(\tilde{x}) \subset X^{\wedge}$, since $X$ is SS there exists a $y \in X_{g}$ such that $x \in U(y)$ and $U(y) \cap N(\epsilon)=\square$. It follows that $\epsilon \geq \epsilon_{y} \subset I(\tilde{x})$. So the set $\left\{\epsilon_{y}: y \leq x, y \in X_{g}\right\}$ is a cofinal subset of $I(\tilde{x})$. Also, note that $G^{\wedge}\left(\epsilon_{y}\right)=\operatorname{inv} \lim \left\{G(u)^{\wedge}, G_{v u}^{\wedge}: v \leq u \in I\left(\epsilon_{y}\right)\right\} \cong G(y)^{\wedge}$ since $y$ is the smallest element of $I\left(\epsilon_{y}\right)$. If $\delta \in G(y)^{\wedge}$ and $s \in G(z)$, define 


$$
\begin{aligned}
\bar{\delta}(s) & =0 & & \text { if } z \not y, \\
& =\delta\left(G_{y z}(s)\right) & & \text { if } z \geq y .
\end{aligned}
$$

Then $\bar{\delta} \epsilon \mathcal{H}\left(\epsilon_{y}\right)$ and $\bar{\delta}$ is identified with the element $\left\langle\delta{ }^{\circ} G_{y x}\right\rangle_{x \geq y}$ of $G^{\wedge}\left(\epsilon_{y}\right)$. The map $K_{y}$ defined for $\delta \in G(y)^{\wedge}$ by $\delta \rightarrow\left\langle\delta \circ G_{y z}\right\rangle_{z \geq y}$ is the natural iseomorphism of $G(y)^{\wedge}$ onto $G^{\wedge}\left(\epsilon_{y}\right)$. If $x \geq y \in X_{g}$, then for $\delta \in G(y)^{\wedge}$, we have

Therefore

$$
\begin{aligned}
\hat{G_{\epsilon_{x} \epsilon_{y}}} \circ K_{y}(\delta) & =\hat{G_{\epsilon_{x} \epsilon_{y}}}\left(\left\langle\delta \circ G_{y u}\right\rangle_{u \geq y}\right)=\left\langle\delta \circ G_{y v}\right\rangle_{v \geq x} \\
& =\left\langle\delta \circ G_{y x} \circ G_{x v}\right\rangle_{v \geq x}=K_{x}\left(\delta \circ G_{y x}\right)=K_{x} \circ \hat{G_{y x}}(\delta) .
\end{aligned}
$$

$$
\begin{aligned}
& G^{\wedge \wedge}(\tilde{x})=\operatorname{inv} \lim \left\{\left(G^{\wedge}(\epsilon)\right)^{\wedge},\left(\hat{G_{\delta \epsilon}}\right) \hat{:}: \epsilon \geq \delta \in I(\tilde{x})\right\} \\
& \cong \operatorname{inv} \lim \left\{\left(\hat{G}\left(\epsilon_{y}\right)\right) \hat{},\left(\hat{G_{\epsilon_{z}} \epsilon_{y}}\right) \hat{:} y \leq z \leq x \text { and } y, z \in X_{g}\right\} \\
& \cong \operatorname{inv} \lim \left\{\left(G(y)^{\wedge}\right)^{\hat{n}},\left(\hat{G_{y x}}\right)^{\wedge}: y \leq z \leq x \text { and } y, z \in X_{g}\right\} \\
& =\operatorname{inv} \lim \left\{G(y), G_{y z}: y \leq z \leq x \text { and } y, z \in X_{g}\right\} \text {. }
\end{aligned}
$$

Let $\rho_{x}$ be the composition of the above iseomorphisms. Explicitly, $\rho_{x}$ is the map defined from $G^{\wedge \wedge}(\tilde{x})$ into inv $\lim \left\{G(y), G_{y z}: y \leq z \leq x\right.$ and $y, z \epsilon$ $\left.X_{g}\right\}$ as follows, for $\left\langle\sigma_{\epsilon}\right\rangle \in G^{\wedge \dot{x}}(\tilde{x}):\left\langle\sigma_{\epsilon}\right\rangle, \epsilon \in I(\tilde{x}) \rightarrow\left\langle\sigma_{\epsilon_{y}}\right\rangle, y \in X_{g} \rightarrow\left\langle\sigma_{y}\right\rangle$, $y \in X_{g} \rightarrow\left\langle s_{y}\right\rangle, y \in X_{g}$ where $K_{y}\left(\sigma_{y}\right)=\left\langle\sigma_{\epsilon_{y}}\right\rangle$ and where $s_{y} \in G(y), y \in X_{g}$ and $y \leq x$, and with respect to $G(y), \tilde{s}_{y}=\sigma_{y}$.

Let $g \in G(x)$ and $\omega=\left\langle\omega_{y}\right\rangle \in G^{\wedge}(\epsilon), \epsilon \in X^{\wedge}$. Then

$$
\tilde{g}(\omega)=\omega(g)= \begin{cases}0 & \text { if } x \in N(\omega), \\ \omega_{x}(g) & \text { if } x \in I(\omega) .\end{cases}
$$

With respect to $G(x), \omega_{x}(g)=\tilde{g}\left(\omega_{x}\right)$. In particular for $x \geq z, z \in X_{g}$ and $\sigma \in G(y)^{\wedge}, \tilde{g}(\bar{\sigma})=\bar{\sigma}(g)=\sigma\left(G_{z x}(g)\right)=\widetilde{G_{z x}(g)}(\sigma)$ (relative to $G(z)$ ). If $d$ denotes the map $s \rightarrow \tilde{s}$ and $d_{x}: G(x) \rightarrow G^{\wedge \wedge}(\tilde{x}), x \in X$, denotes the restriction of $d$, we can conclude that

$$
\rho_{x} \circ d_{x}=\psi_{x}: G(x) \rightarrow \operatorname{inv} \lim \left\{G(y), G_{y z}: y \leq z \leq x \text { and } y, z \in X_{g}\right\} .
$$

$(\mathrm{A} \Rightarrow \mathrm{B})$ If $d$ is an iseomorphism, then for each $x \in X, d_{x}$ is an iseomorphism which implies that $\psi_{x}=\rho_{x} \circ d_{x}$ is an iseomorphism.

$(\mathrm{B} \Rightarrow \mathrm{A})$ Let $U$ be an open set of $X, x \in X_{g}$, and $V_{x}$ an open set in $G(x)$. Define $W\left(U, V_{x}\right)=p^{-1}(U) \cap\left\{s \in \mathrm{Cl}(X, G): u=p(s) \geq x\right.$ and $G_{x u}(s) \epsilon$ $\left.V_{x}\right\}$. The collection of all sets of the form $W\left(U, V_{x}\right)$ is a basis (since $X$ is SS) for a topology which makes $\mathrm{Cl}(X, G)$ into a continuous-inverse semigroup and because of condition $B$ realizes the topologies of $X$ and $G(x)$, 
$x \in X$. The reader is referred to [5] where the details of similar arguments are given.

For each $x \in X$, we have proven that $\rho_{x} \circ d_{x}=\psi_{x}$. Therefore, $d_{x}=$ $\rho_{x}^{-1} \circ \psi_{x}$ and condition B implies that $d_{x}$ is an iseomorphism. Since for $g$ $\epsilon G(x), x \in X, d(g)=d_{x}(g)$, it follows that $d$ is an algebraic isomorphism. If $U$ is an open set of $X, x \in X_{g}$, and $V_{x}$ an open set of $G(x)$, let $U^{\prime}$ be the image of $U$ under the map $x \rightarrow \tilde{x}: X \rightarrow X^{\wedge \wedge}$ and let $Y_{x}=d_{x}\left(V_{x}\right)$. Then $d\left(W\left(U, V_{x}\right)\right)=W\left(U^{\prime}, Y_{x}\right)$ which by 2.2 is an open set of $\mathrm{Cl}\left(X^{\wedge}, G^{\wedge}\right)$. It follows that $d$ is an open map and since it is also continuous, $d$ is an iseomorphism.

That the topology is unique follows from the observation that in 2.2 the topologies of $S^{\wedge}=\mathrm{Cl}\left(X^{\wedge}, G^{\wedge}\right)$ and $S^{\wedge}=\mathrm{Cl}\left(X^{\wedge \wedge}, G^{\wedge}\right)$ depend upon $X$ and on the functor $G$ and not on the global topology of $S$. Thus the topology on $S^{\wedge \wedge}$ is determined uniquely and hence the topology of $S$ is unique.

Using the preceding theorem and $[2,3.5]$, we have the following

Corollary. If $S$ is a locally compact semigroup, then the map $s \rightarrow \tilde{s}$ : $S \rightarrow S^{\wedge \wedge}$ is an iseomorphism iff $S$ is an abelian continuous-inverse semigroup with identity which has the following two properties:

(i) if $X$ is the semilattice of idempotents of $S, x \rightarrow \tilde{x}: X \rightarrow X^{\wedge}$ is an iseomorphism;

(ii) if $G$ is the associated functor of $S, G(x)=\operatorname{inv} \lim \left\{G(y), G_{z y}\right.$ : $z \leq y \leq x$ and $\left.z, y \in X_{g}\right\}$.

Remark. For $X$ locally compact, Sneperman [10] gives necessary and sufficient conditions for (i) to hold. He also gives a duality theorem for locally compact abelian continuous-inverse semigroups, but he gives a technical condition which appears to correspond to condition (ii).

\section{BIBLIOGR APHY}

1. C. W. Austin, Duality theorems for some commutative semigroups, Trans. Amer. Math. Soc. 109 (1963), 245-256. MR 27 \#3737.

2. A. C. Baker and J. W. Baker, Duality of topological semigroups with involution, J. London Math. Soc. 44 (1969), 251-260. MR 38 \#4596.

3. J. W. Baker and N. J. Rothman, Separating points by semicharacters in topological semigroups, Proc. Amer. Math. Soc. 21 (1969), 235-239. MR 38 \#4598.

4. T. T. Bowman, A construction principle and compact Clifford semigroups, Semigroup Forum 2 (1971), no. 4, 343-353. MR 45 \#2075.

5. - Construction functors for topological semigroups (submitted).

6. A. H. Clifford and G. B. Preston, The algebraic theory of semigroups. Vol. I, Math. Surveys, no. 7, Amer. Math. Soc., Providence, R. I.,1961. MR 24 \#A2627. 
7. R. O. Fulp, Character semigroups of locally compact inverse semigroups, Proc. Amer. Math. Soc. 27 (1971), 613-618. MR 43 \#2148.

8. J. D. Lawson, Topological semilattices with small semilattices, J. London Math. Soc. (2) 1 (1969), 719-724. MR $40 \# 6516$.

9. L. B. Sneperman, Finite-dimensional repres entations of some compact topological semigroups, Dokl. Akad. Nauk SSSR 176 (1967), 538-540= Soviet Math. Dokl. 8 (1967), 1157-1159. MR 36 \#2732.

10. - On the theory of characters of locally bicompact topological semigroups, Mat. Sb. 77 (119) (1968), 508-532 = Math. USSR Sb. 6 (1968), 471-492. MR 38 \#5984.

DEPARTMENT OF MATHEMATICS, UNIVERSITY OF FLORIDA, GAINESVILLE, FLORIDA 32601 